\title{
STUDY OF ANTIBIOTIC SENSITIVITY PATTERN AMONG COMMUNITY ACQUIRED PNEUMONIA PATIENTS AT AN OUTPATIENT SETTING IN A TERTIARY CARE CENTRE
}

\author{
Kodur Ramamurthy Raveendra1, Jayachandra², Dwijaraj Hegde 3 \\ 1 Professor, Department of Medicine, Bangalore Medical College and Research Institute. \\ ${ }^{2}$ Associate Professor, Department of Medicine, Bangalore Medical College and Research Institute. \\ 3Post Graduate, Department of Medicine, Bangalore Medical College and Research Institute.
}

\begin{tabular}{l}
\hline ABSTRACT \\
\hline BACKGROUND \\
Community Acquired Pneumonia (CAP) patients are being treated with empirical antibiotics by best guess method by the \\
clinician, especially at outpatients. MDR strains are being reported from c/s reports and Gram negative rods are fast increasing \\
both in the aetiology and mortality of CAP patients.
\end{tabular}

\section{AIM OF STUDY}

To find out the antibiotic usage, sensitivity pattern and treatment outcome among CAP patients attending Medicine OPD at a tertiary hospital.

\section{MATERIALS AND METHODS}

188 CAP patients treated on outpatient basis with empirical antibiotics between Jan. and Dec. 2013 at Victoria Hospital, Bangalore, were included in this cross-sectional study. All relevant investigations including sputum c/s were done.

\section{RESULTS}

Positive culture reports were obtained in 104 (55.31\%) out of 188 patients. Following are the isolates - Strep. pneumoniae 42.30\%, Klebsiella 9.61\%, Staph. aureus 11.53\%, Pseudomonas 7.69\%, E. coli 1.92\%, H. influenza 3.84\%, Proteus spp. 3.84\% and mixed growth in 3.84\% of patients; $48.93 \%$ outpatients of CAP received Amoxicillin and $10.63 \%$ of patients received Ciprofloxacin, Amoxiclav and Azithromycin respectively as empirical antibiotics. More than 50\% resistance was seen for Amoxicillin, Amoxiclav, Azithromycin, Ciprofloxacin, Doxycycline and Ceftriaxone for streptococcus species. Similar resistance pattern was seen for other organisms.

\section{CONCLUSION}

This study highlights the importance of culture tests and selection of proper antibiotics and to avoid misuse and abuse of higher antibiotics to prevent the emergence of MDR strains. An antibiotic policy at every level may help.

\section{KEYWORDS}

CAP, Empirical Therapy, Drug-Resistance, MDR Strains.

HOW TO CITE THIS ARTICLE: Raveendra KR, Jayachandra, Hegde D. Study of antibiotic sensitivity pattern among community acquired pneumonia patients at an outpatient setting in a tertiary care centre. J. Evolution Med. Dent. Sci. 2016;5(54):3636-3639, DOI: $10.14260 /$ jemds/2016/838

\section{INTRODUCTION}

Antibiotics have saved millions of lives across the globe from the time of their invention. Today, we cannot imagine a day without antibiotics. Unfortunately, today due to the overuse, abuse and misuse of antibiotics, resistant bacteria have surfaced to cause increased mortality and morbidity. In the battle between microbes and antibiotics, the microbes have often won many battles as the resistance pattern has travelled from simple drug resistance to Multidrug Resistance (MDR) to Total Drug Resistance (TDR).

Community Acquired Pneumonia (CAP) is one of the most common infection that brings patients to the hospital. Pneumonia is still the number one infectious cause of death in the United States. ${ }^{1}$ Appropriate antibiotic therapy is the first important step in the treatment of CAP as early

Financial or Other, Competing Interest: None.

Submission 24-05-2016, Peer Review 19-06-2016,

Acceptance 25-06-2016, Published 07-07-2016.

Corresponding Author:

Dr. Kodur Ramamurthy Raveendra,

Professor, Department of Medicine,

Bangalore Medical College and Research Institute,

K. R. Road, Bangalore-560002.

E-mail: drkrraveendra@gmail.com

DOI: $10.14260 /$ jemds/2016/838 administration of antibiotics is seen to decrease the mortality among CAP patients. ${ }^{2}$ The selection of the antibiotic for the outpatient is usually 'empirical' or by the 'best guess' method and varies from place-to-place and from consultant-toconsultant as there are no governing laws towards the rational use. ${ }^{3} \mathrm{~A}$ few guidelines like American Thoracic Society (ATS) and British Thoracic Society (BTS). ${ }^{4}$ are always helpful in this regard and a decrease in mortality has been documented with the implementation of guideline-based management. 5 The choice of the appropriate antibiotic therapy depends upon the likely pathogen, the antibiotic resistance pattern in the community, history of antibiotic usage and the co-morbid conditions. The recommended duration of therapy is usually for 5 to 7 days based on the clinical response. ${ }^{6}$ The choice of antibiotics is further complicated by the emergence of MDR strains in the community, poor or non-responders, improper or inadequate use of antibiotics, poor follow-up with culture reports and the presence of multiple risk factors.

\section{AIM OF THE STUDY}

- To know the antibiotic usage and sensitivity pattern in CAP patients treated as out-patients. 
- To know the treatment outcome for the empirical antibiotics used for CAP patients on an out-patient basis.

\section{MATERIALS AND METHODS}

The study was designed as a hospital-based cross-sectional study conducted at Victoria Hospital, a Tertiary Care Centre at Central Bangalore, attached to BMCRI, from 01 January 2013 to 30 June 2014, for a period of one and a half years. A total of 296 patients (186 males and 110 females) attending medical OPD with acute chest symptoms were screened and selected after chest X-ray confirmation of pneumonia. All these patients were clinically stable and did not require hospitalization and patients accepted to take oral antibiotic therapy. In 268 patients, sputum was sent for gram staining and culture-sensitivity investigations. All were started with antibiotics (Different antibiotics by different consultants), mainly based upon the availability of antibiotics in the hospital (Government supply) and the purchasing capacity of patients. Out of 268 patients, 188 returned after 3 days (3-7 days) with sputum culture and sensitivity reports. Culture positivity was seen in 104 patients. A detailed work-up was done for this group of patients of 188 (Detailed history regarding antibiotic use, misuse, co-morbid conditions and risk factors and other relevant investigations) regarding antibiotic sensitivity and response and treatment outcome.

\section{RESULTS}

A total of 188 patients $(\mathrm{n}=188)$ who came with sputum C/S reports after 3-5 days of screening visit were included in the study. Of the 188 patients, 116 were males and 72 patients were females with a Male:Female ratio of 1:06. Out of the 188 patients culture was positive in 104 patients, 68 being male and 36 being female. Culture positivity was seen in 40 patients in the age group of 31-40 yrs., 24 patients in the age group of 41-50 yrs. and 18 patients in the age group of 21-30 yrs. The common antibiotics prescribed as empirical treatment for CAP patients on outpatient basis includeAmoxicillin in 92, Ciprofloxacin in 20, Ofloxacin in 4, Amoxiclav in 20, Azithromycin in 20, Cefixime in 10, Levofloxacin in 8, Clarithromycin in 6 and others in 8 patients. On chest X-ray-68 had Right lower lobe consolidation, 44 had Left lower lobe consolidation, 40 had Right upper lobe consolidation, 24 had Left upper lobe consolidation, 8 had multi-lobar consolidation and 4 patients had Right middle lobe consolidation.

Most common symptoms were - fever (96\%), cough (92\%), breathlessness (94\%), chest pain (60\%), fatigue (40\%) and haemoptysis (6\%). Important co-morbid conditions include Cigarette smoking (28\%), COPD (18\%), Diabetes (16\%), Hypertension (16\%), Anaemia/Malnutrition (12\%), Bronchial Asthma (3\%), IHD (2\%) and HIV-positive status (2\%). Single risk factor was noticed in 84 patients, 2 risk factors in 66 patients, 3 risk factors in 32 patients and $>3$ risk factors in 24 patients.

48 out of 188 patients were exposed to one antibiotic (10 have completed the full course) before coming to the present consultation and 12 were exposed to 2 antibiotics; 16 of 188 were frequent antibiotic users (Mainly self-medication from across the counter).

Of 188 patients 104 patient's sputum culture was positive and the organisms isolated were-Streptococcus pneumonia in 44 , other Streptococcus species in 16, Staphylococcus aureus in 12, Klebsiella spp. in 10, Pseudomonas aeruginosa in 08 , Proteus species in 04, H. influenza in 04, E. coli in 02 and others in 04 patients.

After the sputum C/S report, a change in antibiotics was required in 44 patients ( 30 oral and 14 injectables); 18 patients due to persistence of symptoms and want of intravenous antibiotics were referred for admission ( 2 patients were admitted for DKA). On analysis of $\mathrm{C} / \mathrm{S}$ reports, resistance to 1 class of antibiotics was seen in 88 patients, 2 class of antibiotics in 64, 3 class of antibiotics in 24 and more than 3 in 08 patients. Among 84 culture negative patients 56 patients showed improvement with empirical antibiotics, 20 patients required another antibiotic (Azithromycin/Clarithromycin/Doxycycline); 7 patients tested positive for HIV serology, 4 patients for H. influenzae and 3 for Mycoplasma and urine antigen positive for Legionella spp. in 2 patients; 8 patients required hospitalization for worsening of symptoms.

Resistance to streptococcus pneumoniae was seen in $45.45 \%$ of patients for amoxiclav, $81.18 \%$ for Amoxicillin, $63.62 \%$ for azithromycin and Cefuroxime, $75 \%$ for ciprofloxacin, $63.62 \%$ for doxycycline and $50 \%$ for ceftriaxone and Cefoperazone. Resistance to Staph. aureus was noted in $75 \%$ of patients for Amoxiclav, $83.33 \%$ for Amoxicillin and Azithromycin, 50\% for Ciprofloxacin and Ofloxacin, $66.66 \%$ for Cefixime, Doxycycline, Levofloxacin and Cefuroxime. Resistance to Klebsiella species was found to be $80 \%$ for amoxiclav, $70 \%$ for gentamycin, $30 \%$ for amikacin, $50 \%$ for ciprofloxacin and $60 \%$ for ceftriaxone. Resistance to pseudomonas was observed in $75 \%$ for ciprofloxacin, $25 \%$ for amikacin, $62.50 \%$ for ceftriaxone, $25 \%$ for netilmicin, $37.50 \%$ for piperacillin and meropenem. Resistance to Vancomycin, Linezolid, Levofloxacin, Meropenem and to the other third generation Cephalosporins were observed in 08 to $26(7.7 \%-25 \%)$ of 104 culture positive patients.

\begin{tabular}{|c|c|c|c|}
\hline $\begin{array}{c}\text { Sl. } \\
\text { No. }\end{array}$ & $\begin{array}{c}\text { Organism } \\
\text { Isolated }\end{array}$ & Total & Percentage \\
\hline 1. & Streptococcus Pneumonia & 44 & 42.30 \\
\hline 2. & Other Streptococci Species & 16 & 15.38 \\
\hline 3. & Staphylococci Aureus & 12 & 11.53 \\
\hline 4. & Klebsiella Species & 10 & 9.61 \\
\hline 5. & Pseudomonas Species & 08 & 7.69 \\
\hline 6. & Proteus Species & 04 & 3.84 \\
\hline 7. & H Influenza & 04 & 3.84 \\
\hline 8. & E Coli & 02 & 1.92 \\
\hline 9. & Others (Mixed Growth) & 04 & 3.84 \\
\hline \multicolumn{4}{|c|}{ Chart 1: Organism Isolated on Culture } \\
\hline
\end{tabular}

\begin{tabular}{|c|c|c|c|}
\hline Sl. No. & Antibiotics Used & Total & Percentage \\
\hline 1. & Amoxicillin & 92 & 23.40 \\
\hline 2. & Ciprofloxacin & 20 & 10.63 \\
\hline 3. & Amoxiclav & 20 & 10.63 \\
\hline 4. & Azithromycin & 20 & 10.63 \\
\hline 5. & Cefixime & 10 & 5.31 \\
\hline 6. & Levofloxacin & 08 & 4.25 \\
\hline 7. & Clarithromycin & 06 & 3.19 \\
\hline 8. & Ofloxacin & 04 & 2.12 \\
\hline 9. & Cotrimoxazole & 04 & 2.12 \\
\hline 10. & Others & 04 & 2.12 \\
\hline \multicolumn{4}{|c|}{ Chart 2: Different Antibiotics used Empirically } \\
\hline
\end{tabular}




\begin{tabular}{|c|c|c|c|c|c|c|c|c|}
\hline $\begin{array}{l}\dot{z} \\
\dot{m} \\
\dot{m}\end{array}$ & 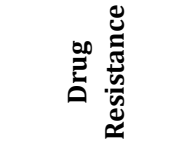 & $\begin{array}{l}\dot{1} \\
\text { Dे } \\
\text { के }\end{array}$ & 㝴 & $\frac{\dot{0}}{\frac{0}{2}}$ & 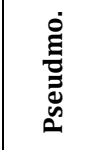 & $\begin{array}{l}\dot{\Xi} \\
\stackrel{\Xi}{\Xi} \\
\dot{x}\end{array}$ & 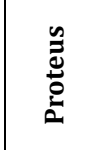 & : \\
\hline 1. & Amoxiclav & 45.45 & 75 & 80 & & & & \\
\hline 2. & Amoxicillin & 81.18 & 83.33 & & 75 & 81.18 & 81.18 & 83.33 \\
\hline 3. & Azithromycin & 63.62 & 83.33 & & & & & \\
\hline 4. & Cefuroxime & 63.62 & 66.66 & & & & & \\
\hline 5. & Cefixime & 63.62 & 66.66 & & 81.18 & & & \\
\hline 6. & Ciprofloxacin & 75 & 50 & & 75 & 50 & & 25 \\
\hline 7. & Doxycycline & 63.62 & 66.66 & & & & & \\
\hline 8. & Ceftriaxone & 50 & 75 & 60 & 62.50 & & & \\
\hline 9. & Cefoperazone & 50 & & 60 & 70 & & & \\
\hline 10. & Ofloxacin & & 50 & 50 & & & & \\
\hline 11. & Levofloxacin & 63.62 & 66.66 & & & & & \\
\hline 12. & Gentamycin & 81.18 & 75 & 70 & 25 & & 25 & 25 \\
\hline 13. & Amikacin & & & 30 & 25 & 25 & 25 & 18 \\
\hline 14. & Netilmicin & & & 37.50 & 25 & 37.50 & & \\
\hline 15. & Piperacillin & & & 12.50 & 37.50 & 50 & 25 & 25 \\
\hline 16. & Meropenem & & & 25 & 37.50 & 37.50 & 37.50 & 50 \\
\hline \multicolumn{9}{|c|}{$\begin{array}{c}\text { Chart 3: Drug Resistance Pattern to } \\
\text { Different Organisms Isolated }\end{array}$} \\
\hline
\end{tabular}

\section{DISCUSSION}

Mild-to-moderate community acquired pneumonia patients are treated on an outpatient basis only on the empirical therapy and later being followed by the culture sensitivity reports. Hence, it is not possible always to target the pathogen when the choice of initial antibiotic is made. Frequent use (Abuse) of antibiotics has changed the bacteriological profile at different parts of the country. Amoxicillin was used as an initial empirical antibiotic in nearly $49 \%$ of patients in this study, which is on par with other studies like-the initial therapy is directed mainly on Streptococcus pneumonia, as it continues to be the leading cause of CAP. ${ }^{7}$ ATS recommends macrolides as the first choice and BTS recommends Amoxicillin as the first choice followed by Clarithromycin and Doxycycline as alternatives. A respiratory Fluoroquinolone or a beta-lactam plus a macrolide or Amoxicillin-Clavulanate is preferred in the presence of co-morbidites. $7,1,8$ In the present study a few culture negative CAP patients who earlier failed to respond to empirical antibiotics, later responded to Clarithromycin/Azithromycin/Doxycycline. ATS statement describes the possibility of atypical pathogens (C. pneumoniae, M. pneumonia and L. pneumophila) either infecting or co-infecting with CAP and therefore recommends therapy to account for this possibility. ${ }^{1}$

Pulmonary Tuberculosis is of great concern and fluoroquinolones usage has some limitations in CAP patients. ${ }^{9}$ In the present study, fluoroquinolones were prescribed in $17 \%$ of patients despite a threat of masking PTB symptoms or a chance of fluoroquinolone resistant state in future.

According to Baudouin SV, a specific pathogen could not be isolated in $30 \%$ of CAP patients. ${ }^{10}$ In the present study, culture positivity was seen in $38.8 \%$ (104 out of 268). According to Neuhaus and Ewigs, about $10 \%$ of CAP patients treated on out-patient basis eventually required ICU admission. ${ }^{11}$ In our study, $17.3 \%$ of culture positive and $9.5 \%$ of culture negative CAP patients on empirical antibiotics required admission to the wards for IV antibiotics and 4 patients ( 2 from each group) were treated in medical ICU.
In the present study, $57.7 \%$ of patients responded to the first empirical antibiotic and the remaining $42.3 \%$ required a change in the antibiotics after c/s report. Culture negative patients repeatedly took multiple antibiotics and changed antibiotics frequently; $23.8 \%$ of them required a change in antibiotics due to poor response to initial antibiotics and 9.5\% required admission for further investigations and IV antibiotics. Routine antibiotic coverage for atypical pathogens causing CAP is controversial as many are self-limiting. Udwadia et al, in their study reported Chlamydia pneumonia and Mycoplasma pneumonia as the common atypical pathogens causing CAP. ${ }^{12}$ This will explain for those patients who failed to respond to empirical antibiotics, but later responded to Azithromycin or Clarithromycin or Doxycycline.

Among culture positive patients, resistance to multiple drugs including for higher antibiotics were noted for common organisms. Streptococcus pneumonia was the commonest organism and more than 50\% showed resistance to Amoxicillin, Azithromycin, Ceftriaxone, Cefuroxime, Cefoperazone. Resistance for Klebsiella was also high - more than 50\% was observed for Amoxiclav, Ceftriaxone, gentamycin and Ciprofloxacin. Resistance to Staph. aureus, was observed in more than $50 \%$ for Amoxicillin, Amoxiclav, Azithromycin, Ciprofloxacin, Ofloxacin, Levofloxacin and Cefixime. Resistance to other organisms like pseudomonas, $\mathrm{H}$ Influenza, E Coli were also high and the newer antibiotics are not spared; $42.32 \%$ of S. pneumoniae, $25 \%$ Klebsiella, 38.4\% Pseudomonas, $17.3 \%$ S. aureus were resistant to more than three antibiotics. Thus, multi-drug resistant strains are common, especially in patients who frequently took antibiotics.

The most practiced guidelines for the treatment of CAP include ATS. ${ }^{1}$ and BTS. ${ }^{4}$ and from Indian perspective is from PGIMER. ${ }^{13}$ Chandigarh. Hence, it is better to adhere to some guidelines than best guess methods to prescribe antibiotics. Early administration of appropriate antibiotics pending culture sensitivity report is probably best mode of treatment of a CAP patient in our busy OPDs.

\section{CONCLUSION}

Management of patients with CAP on an outpatient basis is often challenging and taxing. Many may subside on their own like viral pneumonias and many may complicate in due course requiring hospitalization. So initial empirical antibiotic prescription is the single most aspect in the management of CAP patients on an outpatient basis. Knowing the likely organism, hospital antibiograms, patient's risk factors/co-morbid conditions often help to select the initial antibiotic. ATS, BTS, PGIMER guidelines help us to select the initial antibiotic and then to choose second and third option. Unfortunately, the changing bacterial flora and drug resistance pattern (MDR varieties) in the community makes a huge difference in the treatment outcome. Antibiotic misuse and abuse (Irrational prescriptions from Doctors and selfbuying from patients across the counter) has complicated the whole issue. It is high time to have a policy in the antibiotic usage at different levels - hospital, district, state and country to prevent the emergence of MDR strains. Research in the antibiotic field for new antibiotics is as important as to preserve the existing antibiotics. Antibiotic holiday, Antibiotic reserve and hierarchy in the prescription pattern may be some initiatives required from administration to save our patients in near future from multidrug resistant organisms. 


\section{REFERENCES}

1. Mandell LA, Wunderink RG, Anzueto A, et al. Infectious diseases society of America/American thoracic society consensus guidelines on the management of CAP in adults. CID 2007;44(Suppl 2):S27-72.

2. Houck PM, Bratzler DW, Nsa W, et al. Timing of antibiotic administration and outcomes for medicare patients hospitalized with CAP. Arch Intern Med 2004;164(6): 637-44.

3. Philips I. Prudent use of antibiotics: are our expectations justified. Clin Infect Dis 2001;33(Suppl 3):S130-2. doi: 10.1086/321838.

4. Lim WS, Baundouin SV, George RC, et al. BTS guidelines for the management of CAP in adults: update 2009. Thorax 2009; 64(Suppl 3):1-55.

5. Dean NC, Silver MP, Bateman KA, et al. Decreased mortality after implementation of a treatment guideline for community acquired pneumonia. Am J Med 2001;110(6):451-7.

6. Li JZ, Winston LG, More DH, et al. Efficacy of short course antibiotic regimens for CAP: a meta-analysis. Am J Med 2007;120(9):783-9.
7. Fine MJ, Smith MA, Carson CA, et al. Prognosis and outcomes of patients with CAP a meta-analysis. JAMA 1996;275(2):134-41.

8. Arnold FW, Summersgill JT, Lajoie AS. A worldwide prospective of atypical pathogens in CAP. Am J Respir Crit Care Med 2007;175(10):1086-93.

9. Singh A. Fluoroquinolones should be the first line antibiotics to treat CAP in areas of TB in endemicity. Clin Infect Dis 2007;45(1):133.

10. Baudouin SV. The pulmonary physician in critical care 3: critical care management of CAP. Thorax 2002;57(3): 267-71.

11. Athanassa Z, Makris G, Dimopoulos G, et al. Early switch to oral treatment in patients with moderate-to-severe CAP: a meta-analysis. Drugs 2008;68(17):2469-81.

12. Udwadia ZF, Doshi AV, Joshi JM. Aetiology of CAP in India. Eur Respir J 2003;22:5445.

13. Post graduate institute of medical education and research Chandigarh practice. Guideline for management of CAP and HAP. A consensus statement Lung India 2006;23:115-20. 